\title{
EXPLORING THE POTENTIAL OF DETAILED LAND USE VARIABLES IN MODELLING EXTERNAL - INTERNAL TRIPS
}

\author{
Niloufar Shirani-bidabadi ${ }^{1}$, Michael Anderson ${ }^{2}$ \\ ${ }^{1,2}$ Civil and Environmental Engineering Department, University of Alabama in Huntsville, \\ Alabama, 35899, USA
}

Received 11 September 2019; accepted 2 October 2019

\begin{abstract}
The common methodology for distributing External-Internal (EI) trips is to collect traffic counts at the cordon line of a study area and to use total employment of the internal zone as the factor upon which zone attractiveness can be based. This methodology assumes that trips originating from outside the study area have a destination within the study area which is towards areas that are suitable for employment. However, depending on the type of employment at the destination, the attraction may vary. This paper examines different employment-based which makes the zones attractive for external trips. The paper concludes that the three scenarios in which the land use variables are utilized, indicate more improvement than those without considering the land use mix. A combination of retail employment and the number of different land-uses at the destination was the best possible methodology to determine external trip attraction within a study area. Furthermore, simply switching from total employment to retail employment at the destination location can also improve the accuracy of the travel model.
\end{abstract}

Keywords: external-internal, trips, employment, retail, land use, none-retail.

\section{Introduction}

A region may attract external trips depending on how much more it is more attractive compared to its surrounding regions. accurate estimation of external trips for the region is a key component behind the successful implementation of any well-defined travel demand model. For modelling simplicity, researchers have categorized external trips on the basis of the home locations of the individuals with respect to the modelling region i.e. the study area (Baqueri et al., 2018). A key element of concern, as already mentioned, in these travel demand models is that the non-residents external trips are made by individuals living outside the study area, whereas the primary resources of data collection focus on the individuals living inside the study area. Non-residents external trips are further categorized in the literature on the basis of the destination of the trips. The trips that have the destination within the study area are termed as external-internal (EI) trips while the trips that have both ends outside the study area but crossing through the study area are called as external-external (EE) trips. Furthermore, there is not a direct way to collect travel data for those living outside the community. Which informs on the reason for limited number of studies on external trips.

${ }^{1}$ Corresponding author: nb0040@uah.edu 
Non-residents external trips coming towards a study area seek destinations based on the socioeconomic factors such as the availability of the employment at the destination (for either working, shopping, educational, medical, or other purposes), land use (Van Wee, 2009; Cao, 2010; Mokhtarian and Cao, 2008; Gim, 2013; Gehrke and Clifton, 2016) and road infrastructure (capacity and travel time of the transportation system) (Abedin and Mehrabi, 2019; Abedin et al., 2019). Therefore, understanding the relationship between the land use characteristics and external trips is the key to accurately estimate external trips and ultimately making travel demand models as accurate as possible. EI trips models seek to assign trips from outside the study area to appropriate locations within the study area. However, current modeling practices around the US assign EI trips to locations on the basis of the total employment in the area, regardless of the effect of the type of employment and the variety of the land-uses in the area. This study, however, intends to evaluate if the type of employment, retail or non-retail at the destination location, and the variety of land-uses at the destination contribute to the likelihood of attracting EI trips.

This paper aims to address by describing a case study on Huntsville, AL in which different trip attraction scenarios were examined to determine the combination of employment type and land-use variety that produced the best traffic assignment model. Then, the model was validated and its transferability to other communities within Alabama was checked.

The next section of this paper presents an overview of the research work done on modelling external trips. The following sections present the data collection, methodology and the results obtained. The last section provides a concluding discussion.

\section{Literature Review}

There is limited scientific literature available that explicitly tries to estimate external trips. This is basically because usually, limited information is available about the region outside the study area.

The available literature estimates external trips through external surveys that are performed at the external stations of a study area for a specific time period. The external stations are the gateways used to enter or exit the study area. For modelling EI trips through external surveys, the entry and exit stations for each vehicle are marked through the Bluetooth devices placed at the stations. However, modelling EI trips through external surveys is quite an extensive task as the device needs to be placed at each Traffic Analysis Zone (TAZ) within the study area. This can be quite a cumbersome task because, depending on the study area's size, there can be even hundreds or thousands of such zone. To overcome this issue, researchers have tried to develop models that determine the number and destination of EI trips on the basis of the size and the land use characteristics within the study area like population (Anderson, 2005) and employment opportunities (Qian et al., 2012) respectively.

From modelling perspective, a lot of efforts have been put to estimate the external trip generation (Doustmohammadi et a., $l$ 2016). This is because of two reasons. First, generation data collection is easier because the EE/EI entry/exit points are always limited and related. Second, there is abundant data available that can be used 
in modelling external trip generation. For example, Average Daily Traffic (ADT) (Anderson et al., 2006), highway capacity from where the trips enter the study area (Han and Stone, 2008), heavy traffic on the highway (Khan and Anderson, 2014).

While such richness in the explanatory variables in not difficult to obtain for within the study area, the actual EI trip distribution data is an enormous undertaking). However, accurately determining the destination for TAZ of EI trips is important because they are performed within the region of interest. A limited number of studies that already walked the path tried to address this limited information through oversimplification in either the models or the approach, see for instance (Qian et al., 2012).

For modeling EI trips, a simpler approach has been proposed in the literature where the trips that have at least one end i.e. origin or destination in the study area are combined estimated (Huntsinger and Ward, 2015). Simply put, the study aggregated the external trips that had at least one end in the study area to total trips irrespective of separately accounting for the origin and destination location related to the study area. This approach, however, limits the input in the traffic assignment where the EI and InternalExternal (IE) split is arbitrarily provided. Such a model demonstrated little usability from a practitioners' point of view as these numbers cannot be fed into a travel demand for further modelling and policy tests.

Studies have also explored the relationship between travel and land use, either in magnitude (Gim, 2013; Cao, 2010; Mokhtarian and Van Herick, 2016) or variety of land uses (Cervero and Duncan, 2006; Gehrke and Clifton, 2019; Zhang and Zhao,
2017). However, a more realistic approach is to utilize the detailed land use data available for the study area for modeling EI trips (Baqueri et al., 2019).

As mentioned, there are numerous difficulties encountered in external trips data collection (e.g. they are disruptive, expensive and time consuming). This also affected the variety in the statistical modelling; the focus of modelling attempts is on estimating attraction factors applying regression models. For example, based on Huff probability, (Anderson, 2005) incorporated an economic factor into the multiple linear regression equation for estimating External-External (EE) trip attraction. (Qian et al., 2012) with taking advantage of availability of employment data, developed multiple linear regression and the result revealed that Mining industry and AER (arts, entertainments, recreation services) can be attractive factors for small and medium communities respectively. While multiple linear regression model has been widely used in the literature for estimating external trip attraction, it imposes some limitations like predicting the number of trips below zero. Therefore, some other studies developed more advanced models to estimate external trips. For instance, (Talbot et al., 2011) applied multinomial logit regression using road and demographic data for the purpose of distributing external trips between all external stations. However, the studies show that gravity model has a priority over regression models. In a recent study, Cordera et al., 2018) compared Poisson regression with the gravity model and the result showed that the gravity model, under some restrictions, has a better performance in distributing the trips than a regression model without constraints (Cordera et al., 2018).

\section{ijtte 389}


Therefore, the present study aims to consider the advantages of gravity model to evaluate the relationship between land use types and distribution of E-I trips.

\subsection{Investigating the Association of Land use Mix and Traveling}

some previous studies (Manaugh and Kreider, 2013; Mavoa et al., 2018; Munshi, 2016) used the most relevant built environment representing "Diversity". These indices such as entropy index and dissimilarity index can quantify the land use mix and land use balance respectively (Munshi, 2016). However, the limitation of such indices is that, they need to be normalized. However, there are some extreme cases where these indices cannot behave normally. Therefore, we made new indices as input for modelling trip attraction based on magnitude and mixed land uses. These indices, which are further explained in the methodology section, are robust, providing good measures and easy-to-collect data. Generally, the contribution of this study can be explained in three different points:

1. Modelling EI trip distribution within the study area through land use variables;

2. Making new and data-light indices for both magnitude and mixed land uses;

3. Using the indices as inputs in the gravity model for six different scenarios.

\section{Methodology}

The case study described in this paper focuses on applying different EI trip distribution scenarios to decide if there is any better option for defining the external trip distribution on the basis of total employment. The method compares six different scenarios that were capitalized on both the number of employees in the zone, by type, and the number of different landuses in the zone.

The first scenario examines only the type of employment, to determine if there is a difference in using retail, non-retail or total employment as the distribution factor for attracting EI trips. The total number of EI trips entering the study area is distributed among destination TAZs by considering the ratio of employment for each type in the zone and the maximum value of employment for that type (Total Employment Index (TEI)).Similarly, the Retail employment and Non-retail employment values are used to build the Retail Index (RI) and Non-retail Index(NRI) respectively. See Equation (13). The index is then used for each zone and multiplied by the total number of trips.

Retail value $/($ Max retail $)=R I$

Nonretail value/ (Max Nonretail $)=N R I$

$($ Retail + Nonretail $) /($ Max $($ Retail + Nonretail $))$ $=T E I$

The second scenario, examined in this work, focuses on the combination of employment by type and the impact of multiple land-uses. The values tested are shown in Equation (4-6):

Retail value $/($ Max retail $)+L U /($ Max $L U)=$ RLUI

Nonretail $/($ Max Nonretail $)+L U /($ Max LU $)=$ NRLUI

Retail/(Max Retail) + Nonretail/(Max Nonretail) $+L U /(\operatorname{Max} L U)=T E L U I$ 
Where, LU represents the number of land use type, TE is total employment. R and NR indicate retail and non-retail respectively.

The trip distribution is performed using a gravity-based model (Cascetta et al., 2013). The gravity model is formulated as a function of impedance between origin and destination TAZ which in this case is the travel time (Mishra et al., 2013). The gravity model equation is shown in Eq. 7 .

$T_{i j}^{k}=P_{i}^{k} A_{j}^{k} F_{i j}^{k} / \sum_{j=1}^{n} A_{j}^{K} F_{i j}^{k}$

Where, $T$ is trip for purpose $k$ from zone $i$ to $j, P$ is Production for trip purpose $\mathrm{k}$ in zone $i, A$ represents Attraction for trip purpose $k$ in zone $j$, and $F$ indicates Friction factor for trip purpose $k$ between zone $i$ and $j$.

After modeling, Validation of the methodologies tested was performed by examining the total assignment for the Huntsville MPO model to the traffic counts. The statistical test was performed for each of six scenarios.

\section{Data Collection}

Huntsville metropolitan area is located in Madison County. This study area is quite attractive as competed to its surrounding region. The travel demand model for this county has been developed and maintained by the Huntsville Metropolitan Planning Organization (MPO). The model contains 368 Traffic Analysis Zones (TAZs) that represent the city of Huntsville which was modeled using Citilabs CUBE software.

As expected, many trips in Huntsville are attracted from outside the study area for a variety of purposes. On this premise, this area, therefore, has been selected to investigate the factors influencing the destination for EI trips in Huntsville, AL.

To this end, traffic volume, socioeconomic (employment data for both retail and non-retail), roadway network and origin/ destination data within travel demand model, which was originally validated for 2005, were obtained from the Huntsville MPO and aggregated to TAZ. The landuse data is also obtained from the United States Geographical Survey (USGS) of 2005 to ensure that the data were compatible temporally.

Furthermore, the shape files of land uses were imported to Arc GIS 10.2 and different types of land uses were recognized, however, land-uses that would not be related to an urban area's attraction such as: crop lands, agriculture, deciduous, ever greens and other kinds of forest lands, were not included in the modelling process. Other land use types were combined and categorized to seven categories: commercial services, industrial complexes, mixed urban or builtup land, residential, transportation and communication services. For all TAZs, the numbers of land use entities within each zone were collected. The maximum and the minimum number of land uses were ranged from zero to five, see Fig.1.

As can be seen in Fig 1, the downtown area of Huntsville is quite diverse in terms of the opportunities available there. Furthermore, many areas along major highways indicate diversity with the presence of the majority of the land uses. This is a natural process as many entities located themselves along the major highways for easy accessibility and also increasing relevant footprints. 


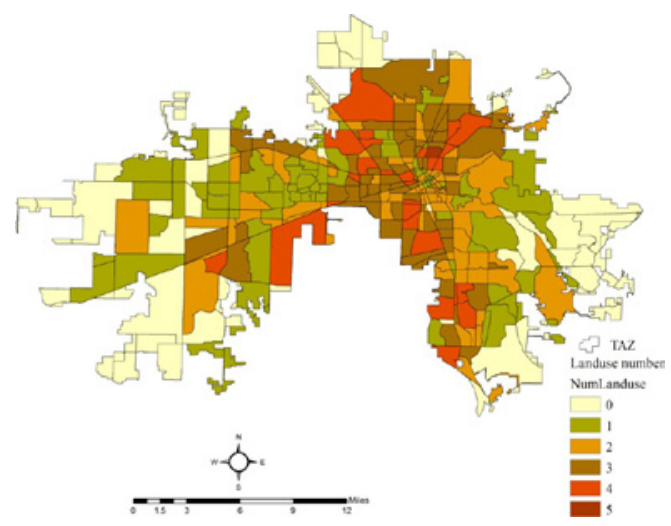

Fig. 1.

The Map of Land use Number of Huntsville's Traffic Analysis Zones

\section{Results}

This section describes the results obtained from fitting the gravity model using the variables described above. As seen in the figure, the major highway on the West attracts the major incoming external traffic in the study area. It feeds the traffic from West such as Decatur, and from South from as far as Alabama and towards the study area. There are fewer external trips generated from the North and East because of the presence of other attractive regions which limits external trips to those regions such as Nashville in North and Atlanta in the East. An interesting observation that can be seen in the figure is that the EI trips from highway 565 located South West of the county can be seen travelling all the way to the downtown area. However, the trips entering the study area from the 72-highway running EastWest are ending before reaching the center of the study area. A quick analysis reveals the region located around the 72 nd highway is quite attractive especially with the presence of the university.

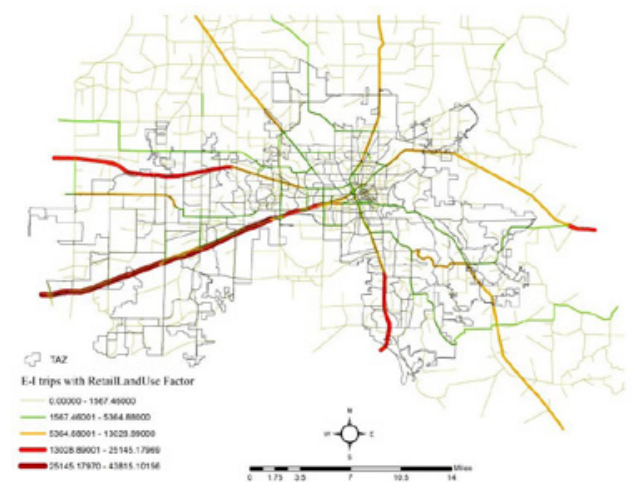

Fig.2.

The E-I Trips Pattern into Huntsville for Non-home Based 
An interesting comparison can be drawn while comparing Fig 1 with Fig 2. Although the majority of hotspots are located along the North-South that passes through the downtown area (Fig 1), this highway attracts less EI trips as compared to the other major highways passing through the study area (Fig 2 ). This conclusion shows that EI trips are not only dependent on the attractiveness of the study area (i.e. the opportunities available to perform the activities) but also on the opportunities available in other locations outside the study area from where these trips are originated.

\subsection{Validation}

EI trips modeling is performed using CUBE 6.4.3. Furthermore, for validation, specific assignments for the EI trips were performed. The validation of the model was performed statistically using 607 traffic counts data across the Huntsville area. These were the same traffic counts used to validate the original travel demand model. For comparison purposes, the modeling that used total employment for the EI trip attractions was the actual model used in 2005.

The total number of EI trips was a little over 143,000 with a total number of trips for the study area just over 1,000,000. For visualization, the pattern of EI trips using "retail+ land use" is shown in Fig. 2. Therefore, it was expected that the different scenarios for EI trip distribution would have different level of accuracy but would be fairly close. The reason behind the close assumption was that the total number of EI trips were assigned slightly less than 15 percent of the total daily trips made in the community. Although the number of trips is very small, they still constitute around $15 \%$ of the total trips which signifies the importance of accurate estimation of external trips up to the level of TAZs and rather than just the origin and destination of external stations.

The statistical test was applied to compare the performance of each factor to predict the EI trips of Huntsville, AL. In order to compare the impact of each scenario for distributing the EI trip factor, the model which was developed with TEI was considered as a base case scenario. Then, the Root Mean Square Error (RMSE) of each scenario was compared to the reference scenario to determine the relative attractiveness of locations. Table 1 shows the RMSE differences (in percent) between each of the five alternatives and TEI .

Table 1

\%RMSE Differences Compared with Base Case

\begin{tabular}{|l|c|c|c|c|c|}
\hline Base Case Scenario: TEI & $\begin{array}{c}\text { Scenario1 } \\
\text { NRI }\end{array}$ & $\begin{array}{c}\text { Scenario2 } \\
\text { RI }\end{array}$ & $\begin{array}{c}\text { Scenario3 } \\
\text { RLUI }\end{array}$ & $\begin{array}{c}\text { Scenario4 } \\
\text { NRLUI }\end{array}$ & $\begin{array}{c}\text { Scenario5 } \\
\text { TELUI }\end{array}$ \\
\hline RMSE Improvement (\%) & -0.22 & 0.34 & 1.05 & 1.01 & 0.97 \\
\hline
\end{tabular}

As expected, the result shows that all the three scenarios in which the land use variables are utilized, indicate more improvement (1.05, 1.01 and 0.97) than those without considering the land use mix $(-0.22$ and 0.34$)$. This signifies the influence of socioeconomic factors in increasing the attractiveness of the study area when applied together with the relative share of land uses such as commercial, transportation and communication, built up, residential, industry and other urban land use services. 
The third scenario (RI, 1.05) shows the best improvement which implies the trips from outside the study area would likely be heading to high-retail-employment locations which provide a large number of land-uses. This indicates that trips are heading to locations with a magnitude of shopping options, but desire variety as well.

\subsection{Sensitivity Analysis}

To further the study and analysis of the results, a sensitivity analysis was performed using only retail and non-retail employment, without land-use, as it saves a variable that is not usually collected. This implementation enhances the application and transferability of the model to other similar regions.

This exercise was performed with the intention to allow for the identification of a maximum point between retail and non-retail that might be optimal, based on the data. For example, if it were to be determined that the reasoning behind an individual selection of) a specific zone for the destination was based on $25 \%$ non-retail employment and $75 \%$ retail employment, an ideal distribution would fall somewhere in-between all retail and all non-retail. The analysis indicated that the best results were obtained when $100 \%$ retail employment, as the attraction factor for EI Trips, was used (confirming the result showed in table 1 , where improvement of RI, 0.34 > NRI, -0.22).

Finally, to check for transferability of the results, the EI trip attraction methodology that used retail employment versus total employment was performed. The transferability was accessed by transferring the model to Montgomery, AL; Gadsden, $A L ;$ and Muscle Shoals/Florence, AL travel demand models. These locations were selected because they represent small and medium-sized communities and are also located within Alabama. The obtained results using retail employment versus total employment for the EI trips attraction factor show that there is an improvement of 3-6 percent in RMSE for different communities (Table 2).

Table 2

Transferability and Comparison

\begin{tabular}{|l|c|c|c|}
\hline City & Montgomery & Gadsden & Muscle Shoals \\
\hline RMSE Improvement (\%) & -4.7 & -6.2 & -3.6 \\
\hline
\end{tabular}

\section{Conclusion}

The goal of this study was to examine different scenarios to distribute EI trips for traffic models. With this premise, the trips coming to a study area are not necessarily heading to locations simply based on total employment but may also to retail employment and shopping locations within the study area. This is because these locations also provide a variety of land-use alternatives that ultimately allow travelers to meet their needs in a single location, limiting extra trips. This was proved by the results as the best assignment methodology for the EI trips was obtained when combination of variables, including retail employment and number of land-uses, was used. Additionally, if the inclusion of land-use is not considered in the model, simply switching the attraction parameter for EI trips from total employment to retail employment can improve model results 
(among the scenarios not considering land use mixed). This was shown in the transferability study that included the methodology being implemented for other communities.

\section{Overall, there was not a large difference} shown in different statistical values since the total number of EI trips is not a substantial number of trips compared to the total number of internal trips, however, the marginal improvement of each trip purpose is a contribution and by making individual improvements to each trip purpose, the overall accuracy of the models will be improved. The improvements to the models can assist with the identification and prioritization of capacity projects.

\section{References}

Abedin, M.; Farhangdoust, S.; Mehrabi, A. B. 2019. Fracture detection in steel girder bridges using selfpowered wireless sensors. In Proceedings of the In Risk-Based Bridge Engineering: Proceedings of the 10th New York City Bridge Conference, August 26-27, New York City, USA. 13 p.

Abedin, M. Mehrabi, A. B. 2019. Novel approaches for fracture detection in steel girder bridges, Infrastructures 4(3): 42 .

Anderson, M.D. 2005. Spatial economic model for forecasting the percentage splits of external trips on highways approaching small communities, Transportation Research Record 1931(1): 68-73.

Anderson, M.D.; Abdullah, Y.M.; Gholston, S.E.; Jones, S.L. 2006. Development of a methodology to predict through-trip rates for small communities, Journal of Urban Planning and Development 132(2): 112-114.

Baqueri, S.F.; Adnan, M.; Bellemans, T. 2018. Modeling external trips: Review of past studies and directions for way forward, Journal of Transportation Engineering, Part A: Systems 144(9): 04018051.
Baqueri, S.F.; Adnan, M.; Knapen, L.; Bellemans, T.; Janssens, D. 2019. Modelling distribution of externalinternal trips and its intra-region and inter-region transferability, Arabian Journal for Science and Engineering 44(5): 4517-4532.

Cao, X. 2010. Exploring causal effects of neighborhood type on walking behavior using stratification on the propensity score, Environment and Planning A 42(2): 487-504.

Cascetta, E.; Cartenì, A.; Montanino, M. 2013. A new measure of accessibility based on perceived opportunities, Procedia-Social and Behavioral Sciences 87: 117-132.

Cervero, R.; Duncan, M. 2006. Which reduces vehicle travel more: jobs-housing balance or retail-housing mixing?, Journal of the American Planning Association 72(4): 475-490.

Cordera, R.; Sañudo, R.; dell'Olio, L.; Ibeas, Á. 2018. Trip distribution model for regional railway services considering spatial effects between stations, Transport Policy 67: 77-84.

Doustmohammadi, M.; Anderson, M.D.; Swain, J.J. 2016. Evaluation of trip generation at a free standing discount superstore, International Journal for Traffic and Transport Engineering 6(4): 495 - 502.

Gehrke, S.R.; Clifton, K.J. 2016. Toward a spatialtemporal measure of land-use mix, Journal of Transport and Land Use 9(1): 171-186.

Gehrke, S.R.; Clifton, K.J. 2019. An activity-related land use mix construct and its connection to pedestrian travel, Environment and Planning B: Urban Analytics and City Science 46(1): 9-26.

Gim, T.H.T. 2013. The relationships between land use measures and travel behavior: A meta-analytic approach, Transportation Planning and Technology 36(5): 413-434. 
Han, Y.; Stone, J. R. 2008. Synthesized through-trip models for small and medium urban areas, Transportation Research Record 2077(1): 148-155.

Huntsinger, L.F.; Ward, K. 2015. Using mobile phone location data to develop external trip models, Transportation Research Record 2499: 25-32.

Khan, T.; Anderson, M. 2014. Estimation of through trips using existing traffic counts, International Journal for Traffic and Transport Engineering 4(4): 415-424.

Mishra, S.; Wang, Y.; Zhu, X.; Moeckel, R.; Mahapatra, S. 2013. Comparison between gravity and destination choice models for trip distribution in Maryland. In Proceedings of the Transportation Research Board 92nd Annual Meeting, Washington, DC, USA, 13-17.

Manaugh, K.; Kreider, T. 2013. What is mixed use? Presenting an interaction method for measuring land use mix, Journal of Transport and Land Use 6(1): 63-72.

Mavoa, S.; Boulangé, C.; Eagleson, S.; Stewart, J.; Badland, H.M.; Giles-Corti, B.; Gunn, L. 2018. Identifying appropriate land-use mix measures for use in a national walkability index, Journal of Transport and Land Use 11(1): 681-700.

Mokhtarian, P.L.; Cao, X. 2008. Examining the impacts of residential self-selection on travel behavior: A focus on methodologies, Transportation Research Part B: Methodological 42(3): 204-228.
Mokhtarian, P. L.; Van Herick, D. 2016. Quantifying residential self-selection effects: A review of methods and findings from applications of propensity score and sample selection approaches, Journal of Transport and Land Use 9(1): 9-28.

Munshi, T. 2016. Built environment and mode choice relationship for commute travel in the city of Rajkot, India, Transportation research part D: transport and environment 44: 239-253.

Qian, Z.; Han, Y.; Stone, J. R. 2012. Forecasting external trips in small and medium cities based on local economic context, Procedia-Social and Behavioral Sciences 43: 284293.

Talbot, E.S.; Burris, M.W.; Farnsworth, S. 2011. Estimating through-trip travel without external surveys, Transportation Research Record 2254(1): 104-111.

Van Wee, B. 2009. Self-selection: A Key to a Better Understanding of Location Choices, Travel Behaviour and Transport Externalities?, Transport Reviews 29(3): 279-292.

Zhang, M.; Zhao, P. 2017. The impact of land-use mix on residents' travel energy consumption: New evidence from Beijing, Transportation Research Part D: Transport and Environment 57(2017): 224-236. 